\title{
The diabetic pregnancy and offspring BMI in childhood: a systematic review and meta-analysis
}

\author{
L. H. Philipps • S. Santhakumaran • C. Gale • E. Prior • \\ K. M. Logan • M. J. Hyde • N. Modi
}

Received: 8 February 2011 /Accepted: 8 April 2011 /Published online: 31 May 2011

(C) Springer-Verlag 2011

\begin{abstract}
Aims/hypothesis Offspring of mothers with diabetes are at increased risk of metabolic disorders in later life. Increased offspring BMI is a plausible mediator. We performed a systematic review and meta-analysis of studies examining offspring BMI $z$ score in childhood in relation to maternal diabetes.

Methods Papers reporting BMI $z$ scores for offspring of diabetic (all types, and pre- and during-pregnancy onset) and non-diabetic mothers were included. Citations were identified in PubMed; bibliographies of relevant articles were hand-searched and authors contacted for additional data where necessary. We compared offspring BMI $z$ score with and without adjustment for maternal pre-pregnancy BMI. We performed fixed effect meta-analysis except where significant heterogeneity called for use of a random effects analysis.

Results Data were available from nine studies. In the diabetic group unadjusted mean offspring BMI $z$ score was 0.28 higher (all diabetic mothers vs controls $(95 \%$ CI 0.09, 0.47; $p=0.004$; nine studies; offspring of diabetic mothers $n=927$, controls $n=26,384)$ and with adjustment for maternal prepregnancy BMI, 0.07 higher $(95 \% \mathrm{CI}-0.15,0.28 ; p=0.54$; three studies; offspring of diabetic mothers $n=244$, controls $n=11,206$ ). There was no evidence of a difference in offspring BMI $z$ score in relation to type of diabetes (gestational vs type $1, p=0.95$ ).
\end{abstract}

L. H. Philipps $\cdot$ S. Santhakumaran $\cdot$ C. Gale $\cdot$ E. Prior $\cdot$

K. M. Logan $\cdot$ M. J. Hyde $\cdot$ N. Modi $(\bowtie)$

Section of Neonatal Medicine, Department of Medicine, Imperial College London,

Chelsea and Westminster Campus, 369 Fulham Road,

London SW10 9NH, UK

e-mail: n.modi@imperial.ac.uk
Conclusions/interpretation Maternal diabetes is associated with increased offspring BMI $z$ score, although this is no longer apparent after adjustment for maternal prepregnancy BMI in the limited number of studies in which this is reported. Causal mediators of the effect of maternal diabetes on offspring outcomes remain to be established; we recommend that future research includes adjustment for maternal pre-pregnancy BMI.

Keywords BMI · Diabetes · Gestational diabetes · Infant . Maternal diabetes · Meta-analysis · Obesity - Offspring of diabetic pregnancy $\cdot$ Pregnancy $\cdot$ Systematic review

\section{Abbreviations \\ GDM Gestational diabetes mellitus \\ ODM Offspring of diabetic mothers}

\section{Introduction}

The prevalence of maternal diabetes during pregnancy has increased over the last two decades, with every indication that this rise will continue. In 2004, five pregnancies per 1,000 in the UK were complicated by pre-existing diabetes, an increase of more than $50 \%$ since 1996, reflecting a sixfold increase in type 2 diabetes and a $20 \%$ increase in type 1 diabetes [1]. Over the same period the prevalence of gestational diabetes mellitus (GDM) has doubled and currently affects 40 pregnancies per 1,000 [2].

Exposure to a diabetic intrauterine environment has long been recognised as a risk to the fetus. A growing body of evidence suggests that intrauterine exposure to diabetes may 'programme' long-term effects in offspring, as first described in 1979 by Freinkel and Metzger as fuel- 
mediated teratogenesis [3, 4]. Follow-up studies of offspring of diabetic pregnancies have demonstrated an increased rate of diabetes or pre-diabetes in the offspring [5] and features of the 'metabolic syndrome' [6] (obesity, glucose intolerance, hypertension and dyslipidaemia). Animal studies also show that intrauterine exposure to hyperglycaemia increases the risk of overweight, abnormal glucose tolerance and insulin resistance in offspring [7, 8]. Studies in Pima Indians show that the intrauterine environment is an important determinant in the development of diabetes, independent of genetic factors [9]. Children born after their mother developed type 2 diabetes had 3.7 times the risk of developing type 2 diabetes and a higher BMI than siblings born before their mother became diabetic [10]. Although data such as these indicate that a diabetic pregnancy predisposes offspring to obesity and abnormal glucose tolerance independent of genetic determinants [11], the strength of the association and the mediating biological pathways are unknown.

An elevated BMI is a risk factor for the development of type 2 diabetes and often the first marker of the metabolic syndrome [12]. Given the increased risk of developing type 2 diabetes in later life in children born to mothers with diabetes [5], it is plausible that this might be mediated through an increase in offspring BMI. However, to date individual studies examining BMI in offspring of diabetic mothers (ODM) have been small with limited power and contradictory results [13-15].

The primary aim of this systematic review and metaanalysis was to establish the impact of maternal diabetes on offspring BMI $z$ score. Secondary objectives were to distinguish the effect of type of maternal diabetes, and effects after adjustment for maternal BMI.

\section{Methods}

Literature search A systematic review of published studies reporting outcomes of diabetic and non-diabetic pregnancies was undertaken in accordance with the Meta-analysis of Observational Studies in Epidemiology (MOOSE) guidelines for systematic reviews of observational studies [16].

The outcome studied was offspring BMI $z$ score, standardised for age and sex. All types of maternal diabetes mellitus, pre-pregnancy type 1 , type 2 and gestational onset, were considered as exposures. For inclusion, an unselected non-diabetic control group must have been reported within the same paper. Inclusion criteria for offspring were age between 3 and 16 years of age and offspring of a singleton pregnancy.

A search was conducted in PubMed (www.ncbi.nlm.nih. gov) for studies published before 1 November 2010, using
Medline subject heading keywords (Pregnancy in diabetics OR diabetes, gestational) and (Prenatal exposure delayed effects OR Child). The review was limited to human studies published in English. The primary investigator, L. H. Philipps, assisted by E. Prior, conducted the searches and identified studies relevant to the outcome by evaluating the abstract or obtaining a full copy of the paper if no abstract was available. Reference lists of papers retrieved were hand-searched for inclusion in the review. Wherever possible, forward citations of the studies retrieved during the literature search were traced. In studies where anthropometric data on the offspring had been obtained but no BMI $z$ score reported, efforts were made by M. J. Hyde to contact the author to obtain the relevant data. Review articles and commentaries were excluded. Where one or more papers had been published on the same cohort, only the study that reported the outcomes for the cohort at an age closest to the median age of the studies overall was included.

Data extraction and analysis Information on the characteristics of the study population, outcome, exposure, covariates, results and conclusions were independently extracted from each paper by L. H. Philipps, C. Gale and checked by M. J. Hyde and S. Santhakumaran. Study quality was examined in respect to blinding of assessors to maternal diabetes status when measuring offspring outcomes.

A meta-analysis of studies examining the association between diabetes during pregnancy and offspring BMI $z$ score was conducted by L. H. Philipps, S. Santhakumaran and M. J. Hyde in RevMan 5 (5.0.24) [17] using the inverse variance method. Subgroup analysis was carried out to compare offspring BMI $z$ score in relation to type of maternal diabetes. The differences between the subgroup effects were tested for significance using an interaction test [18].

Where studies only reported subgroup analyses for different types of diabetes, pooled means and standard deviations were calculated for use in the meta-analysis of all diabetes types combined, provided that recruitment was not stratified on the basis of type of diabetes, which would prevent the results being generalisable to the population.

A meta-analysis was carried out on studies that reported offspring BMI $z$ score adjusted for maternal pre-pregnancy BMI. Where data adjusted for maternal pre-pregnancy BMI were not available, difference in mean maternal prepregnancy BMI between cases and controls was calculated for each individual study. For each study, the difference in mean maternal BMI was plotted against the mean difference in offspring BMI $z$ score.

A fixed effects analysis was initially carried out for all comparisons. Heterogeneity was assessed using the $\chi^{2}$ test on Cochrane's $Q$ statistic [19] and by calculating $I^{2}$ [20]. If 
significant heterogeneity was present $\left(p<0.05\right.$ from the $\chi^{2}$ test) a random effects meta-analysis was carried out. Potential sources of heterogeneity were investigated by comparing study designs. If study covariates that might explain heterogeneity were identified, meta-regression was used to investigate the significance of the associations. Similarly, where there was doubt as to the eligibility or quality of a study the meta-analysis was performed both with and without the study in order to verify the sensitivity of the conclusions.

Forest plots were used to illustrate study findings and meta-analysis results, and funnel plots to investigate evidence of publication bias. If funnel plots showed asymmetry, Egger's test was performed [21].

\section{Results}

Literature search Using PubMed to perform the literature search, we identified 340 papers, of which 28 matched the inclusion criteria [13, 14, 22-47]. Two additional studies were identified through a search of forward citations [15, 48] and four [49-52] from hand-searching the reference lists of included papers, giving 34 papers in total. Five cohorts were reported in more than one paper, so only the study that reported the outcomes at an age closest to the overall median age of all studies (7 years) was included in the meta-analysis; 10 papers were excluded on this basis [25, 35, 36, 38-40, 42, 47-49]. This resulted in 24 papers that remained for inclusion in the review. A study in Pima Indians was excluded because the control group (mothers who subsequently developed diabetes) was not comparable with other studies [28]. In 16 studies outcome information was not provided in a form suitable for meta-analysis; for example, outcomes were represented as OR for obesity, or height, weight, or BMI, rather than as $z$ scores. We attempted to make contact with the corresponding author and additional data were received for two studies [22, 23]. The authors of 10 studies were not able to provide suitable data, and the authors of four studies did not respond or declined to provide the information requested. Final data suitable for inclusion in the meta-analysis were available for nine studies [13-15, 22, 23, 27, 29, 46, 50]. All were cohort studies (seven prospective and two retrospective). The search strategy is shown in Fig. 1, and a description of included studies is provided in Table 1.

Offspring BMI $z$ score A forest plot of the studies comparing BMI $z$ score in ODM and offspring of nondiabetic mothers is shown in Fig. 2. The pooled mean difference from the fixed effects analysis is $0.21(95 \% \mathrm{CI}$ $0.13,0.28 ; p<0.00001)$. As there is evidence of heterogeneity $\left(p<0.0007 ; I^{2} 70 \%\right)$, a random effects analysis was

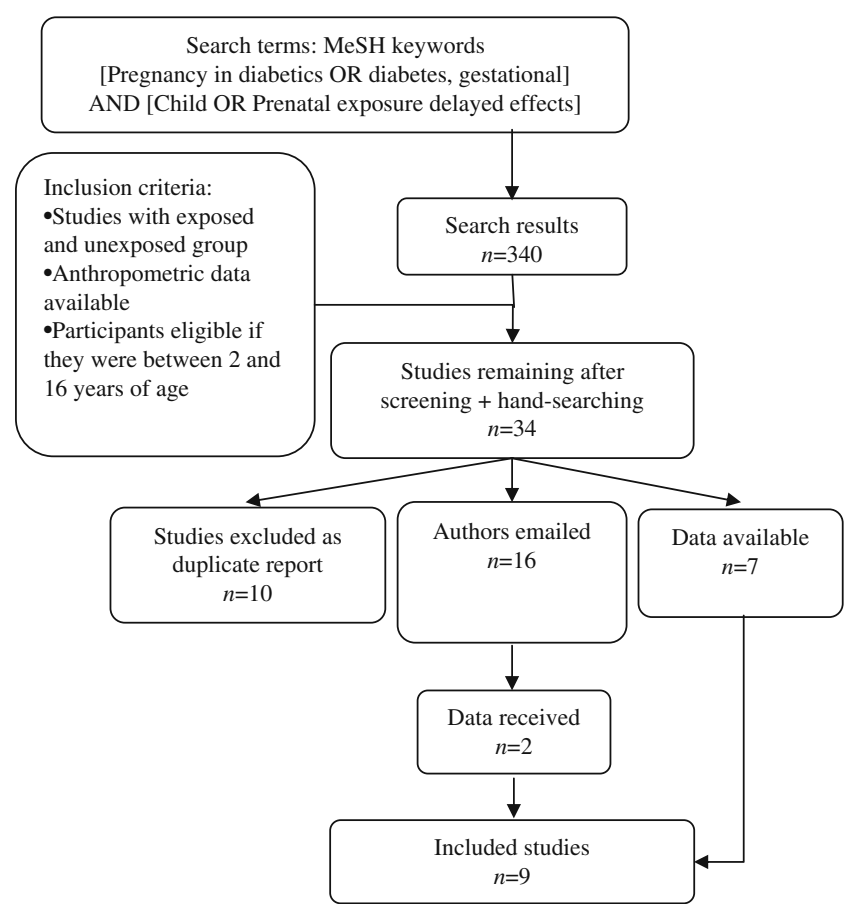

Fig. 1 Flow chart of the search strategy used in this review. The relevant number of papers at each point is given

undertaken giving a pooled estimate of 0.28 (95\% CI 0.09 , $0.47 ; p<0.004)$; the pooled estimate is presented in Fig. 2 .

Subgroup analyses A forest plot of the studies comparing offspring of mothers with gestational-onset diabetes and offspring of non-diabetic mothers is shown in Fig. 3. The pooled mean difference in BMI $z$ score from the fixed effect analysis in this subgroup is 0.20 (95\% CI 0.12, $0.28 ; p<$ $0.00001)$, showing an increase in ODM. There is significant evidence of heterogeneity $(p=0.001)$ and the $I^{2}$ is $76 \%$. When a random effects analysis is performed, the pooled estimate is $0.28(95 \%$ CI $0.05,0.51 ; p=0.02)$; the pooled estimate is presented in Fig. 3.

Data from offspring of mothers with type 1 diabetes (pre-pregnancy) also show an increase in BMI $z$ score compared with control offspring. The pooled mean difference in BMI $z$ score is 0.29 (95\% CI 0.02, 0.55; $p<0.03)$ (Fig. 4). There is no significant evidence of heterogeneity $(p=0.27)$ and the $I^{2}$ is $25 \%$. The BMI difference between the offspring of type 1 diabetic mothers compared with controls is similar to the difference between the offspring of GDM and controls ( 0.29 vs 0.28$)$; an interaction test confirmed that this difference is not significant $(p=0.95)$

Only one study reports a difference between mothers with pre-pregnancy type 2 diabetes and controls [50], so no meta-analysis can be performed for this subgroup. Assessor blinding is not specified in any included study, therefore a subgroup analysis by study quality cannot be performed. 


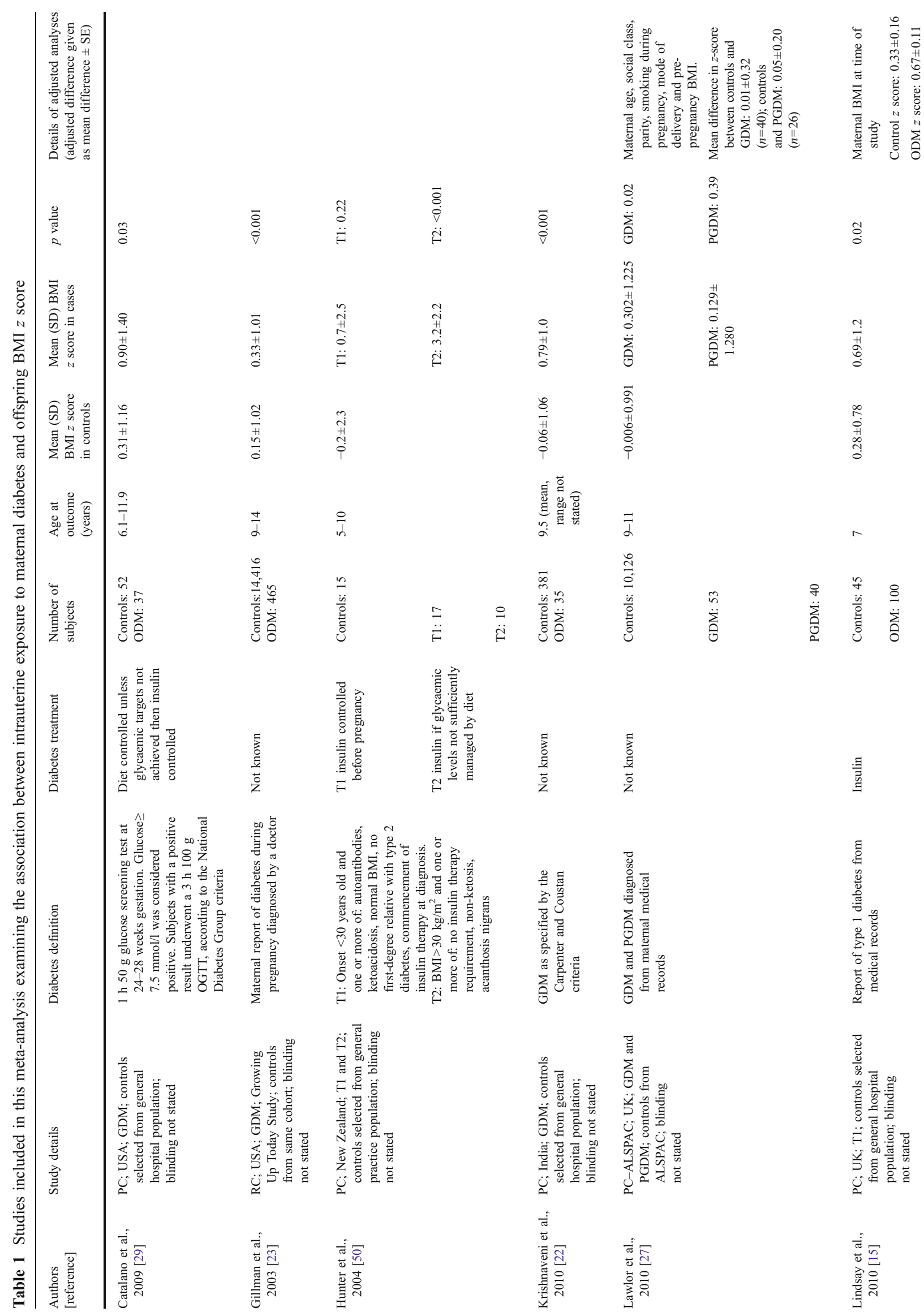




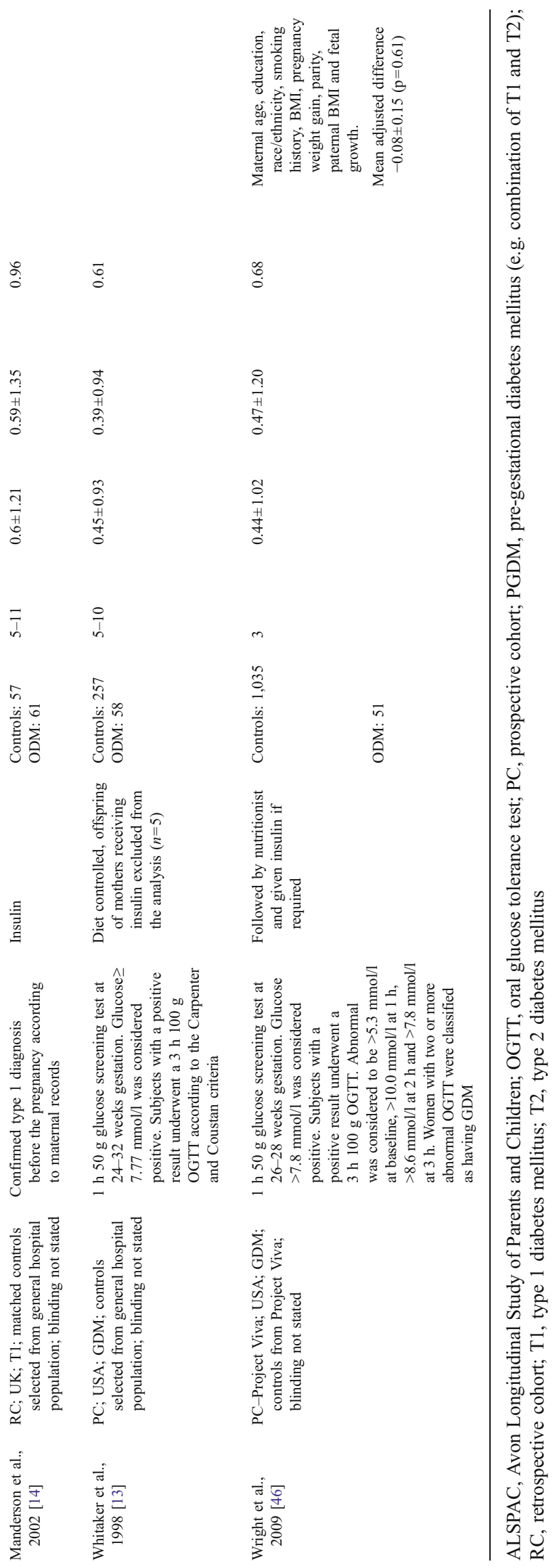




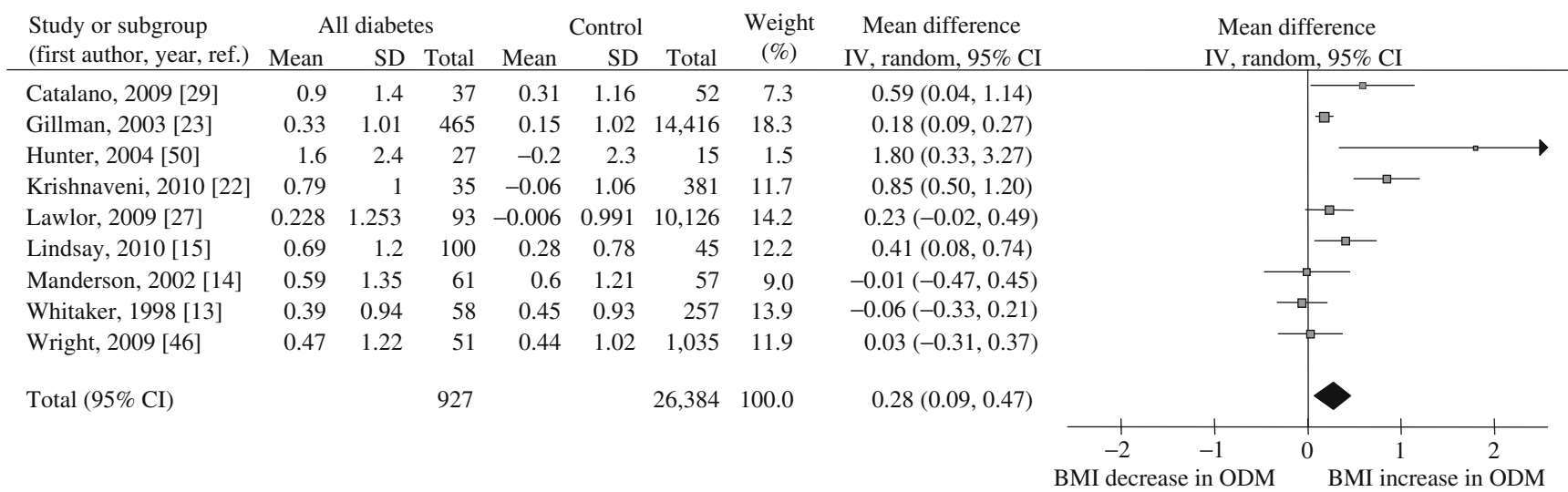

Fig. 2 Forest plot showing the unadjusted association between all types of maternal diabetes and offspring BMI $z$ score. Heterogeneity: $\tau^{2}=0.05$; $\chi^{2}=27.02, d f=8(p=0.0007) ; I^{2}=70 \%$. Test for overall effect: $z=2.90(p=0.004)$. IV, inverse variance; ref., reference

Three studies adjust for covariates including maternal prepregnancy BMI $[15,27,46]$. A meta-analysis of the raw BMI $z$ score data from these three studies shows increased BMI in the offspring of diabetic mothers $(0.23$ [95\% CI 0.06 , $0.40] ; p=0.009 ; I^{2}=20 \%, p=0.29$ ). Using the adjusted data, this effect is attenuated, with a pooled outcome of $0.07(95 \%$ CI $-0.15,0.28 ; p=0.54$ ) (Fig. 5). The heterogeneity of this analysis is not significant $\left(I^{2}=34 \%, p=0.22\right)$.

Figure 6 shows the mean difference in mean maternal pre-pregnancy BMI plotted against the mean difference in offspring BMI $z$ score for five studies presenting these data. Data from Gillman et al. [23] are included, although they did not report pre-pregnancy BMI, but used maternal BMI data at time of the study, approximately 10 years postpartum, on the basis that where available, this was concordant with pre-pregnancy BMI. This analysis suggests that as the difference between the BMI of the diabetic and non-diabetic groups reduces, so too does the difference in offspring BMI $z$ score (Fig. 6). However, a random-effects meta-regression showed this relationship was not significant (increase in mean difference in offspring $z$ score per unit increase in maternal BMI difference $=0.124(95 \%$ CI $-0.08,0.33), p=0.15)$ but this analysis had low power to detect an association as only five studies were included.
A funnel plot of the studies (Fig. 7) shows some asymmetry. Studies lying within the funnel are distributed symmetrically, but there are two studies $[22,50]$ lying to the right of the funnel, indicating potential publication bias towards reporting higher BMI $z$ score in ODM. The Egger test shows no significant evidence for publication bias $(p=0.26)$ but as there are only included studies this cannot be considered conclusive.

\section{Discussion}

This large, comprehensive meta-analysis identifies a strong association between exposure to maternal diabetes in utero and increased offspring BMI in childhood. In studies in which adjustment is made for maternal pre-pregnancy BMI, this relationship is no longer significant. Previous studies have been small with limited power and have provided contradictory results [13-15].

Studies which could not be included in this metaanalysis as they report overweight and obesity as outcomes rather than BMI $z$ score, also show attenuation towards the null following adjustment for maternal prepregnancy BMI. Hummel et al. [51] reported odds ratios for overweight of $1.1(0.7,1.9)$ (unadjusted) and $0.8(0.5$,

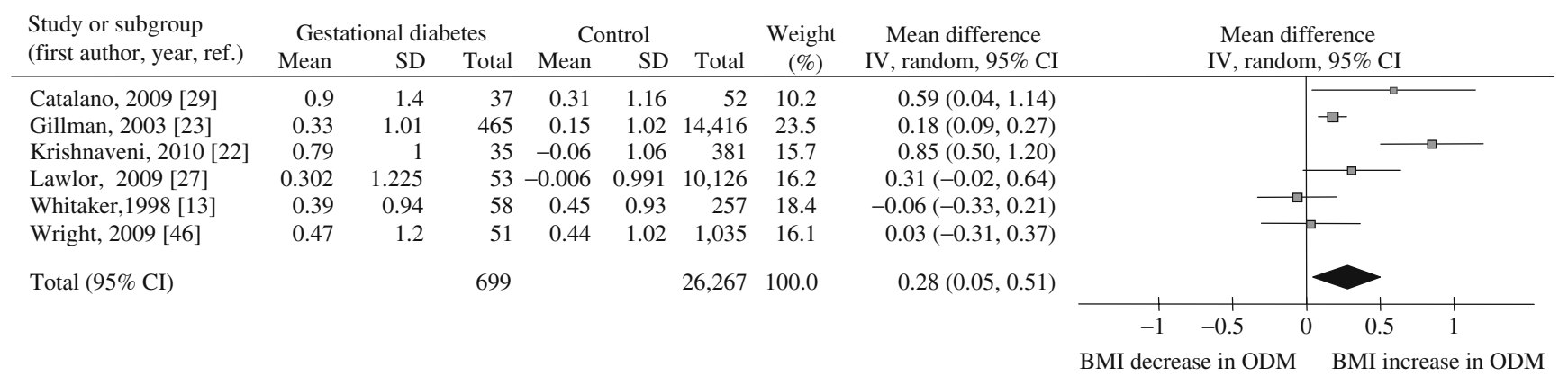

Fig. 3 Forest plot showing the unadjusted pooled analysis of offspring BMI $z$ score of mothers with gestational diabetes mellitus and controls. Heterogeneity: $\tau^{2}=0.06 ; \chi^{2}=25.54, d f=5(p=0.001) ; I^{2}=76 \%$. Test for overall effect: $z=2.39(p=0.02)$. IV, inverse variance; ref., reference 


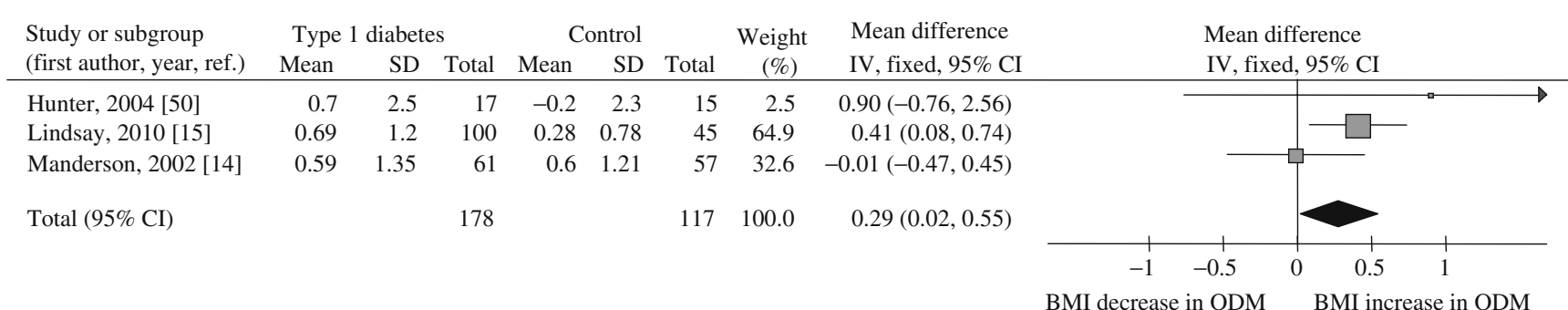

Fig. 4 Forest plot showing the unadjusted pooled analysis of offspring BMI $z$ score of mothers with pre-pregnancy type 1 diabetes and controls. Heterogeneity: $\chi^{2}=2.65, d f=2(p=0.27) ; I^{2}=25 \%$. Test for overall effect: $z=2.12(p=0.03)$. IV, inverse variance; ref., reference

1.4) (adjusted), Gillman et al. [23] $1.4(1.0,1.9)$ (unadjusted) and $1.2(0.8,1.7)$ (adjusted) and Whitaker et al. [13] 1.36 ( $p=0.4$ ) (unadjusted), and $p=0.53$ after adjustment. We also show a correlation between the difference in mean BMI of diabetic and non-diabetic mothers and the difference in mean offspring BMI $z$ score. These data might be interpreted as indicating that the relationship between maternal and offspring BMI in childhood is due to a common genetic potential and/or a shared postnatal obesogenic environment, rather than intrauterine programming secondary to maternal glycaemia of lesser degree than in overt diabetes (for which maternal BMI is a proxy). However, data from studies of Pima Indian siblings show that offspring born before the onset of maternal diabetes have lower childhood BMI than siblings born after the onset of maternal diabetes (with no difference relating to the onset of paternal diabetes) [10]. This suggests the long-term influence of a diabetic intrauterine environment, rather than the impact of postnatal or genetic factors. Similarly, altered offspring glucose tolerance can be induced in rats by intravenous glucose administration during the final days of gestation [7]. Deierlain et al. suggest that blood glucose concentration at 27 weeks gestation is a strong predictor of offspring overweight at 3 years of age, even when adjusted for maternal BMI [53]. Consequently, we suggest that caution should be exercised in rejecting the possibility of intrauterine programming. Adjustment for maternal pre-pregnancy BMI may also mask a potential synergistic relationship with maternal diabetes along a common pathway of hyper- glycaemia during fetal development [27, 54]. This underlines the need for well-designed studies to examine the effects of maternal BMI and diabetes, and the interrelationship between them on offspring.

Human and animal studies show that maternal diabetes induces fetal hyperglycaemia, islet cell hypertrophy and beta cell hyperactivity, all of which may result in fetal hyperinsulinaemia and excess growth, particularly at insulin-sensitive sites such as adipose tissue $[25,55]$. In keeping with this, fetal hyperinsulinaemia shows a positive correlation with childhood overweight [56], even in the absence of macrosomia [35, 57].

Rodent models of intrauterine exposure to hyperglycaemia result in hypothalamic malformations in regions that normally produce orexigenic neuropeptides, including neuropeptide $\mathrm{Y}$, and are associated with hyperphagia and obesity in later life [8]. These data would support the suggestion that in humans, a causal pathway might involve hyperglycaemia-induced modification of hypothalamic appetite regulation, hyperphagia and leptin resistance in the offspring [49]. Maternal overweight/obesity is associated with maternal hyperglycaemia and insulin resistance [58, 59], even when the clinical criteria of GDM are not fulfilled. Pre-pregnancy overweight combined with GDM conveys a greater risk of offspring obesity than exposure to either GDM or pre-pregnancy overweight alone [54]. These data indicate that maternal obesity appears to exaggerate the metabolic abnormalities present in GDM [60], also suggesting that hyperglycaemia, irrespective of aetiology, is the determining mediator.

$\begin{array}{lcrrrrr}\begin{array}{l}\text { Study or subgroup } \\ \text { (first author, year, ref.) }\end{array} & \text { Mean difference } & \text { SE } & \begin{array}{c}\text { Weight } \\ (\%)\end{array} & \begin{array}{l}\text { Mean difference } \\ \text { IV, fixed, 95\% CI }\end{array} & \text { Mean difference } \\ \text { IV, fixed, 95\% CI }\end{array}$

Fig. 5 Forest plot showing the adjusted association between all types of maternal diabetes and offspring BMI $z$ score. Heterogeneity: $\chi^{2}=$ $3.02, d f=2(p=0.22) ; I^{2}=24 \%$. Test for overall effect: $z=0.61(p=0.54)$. IV, inverse variance; ref., reference 


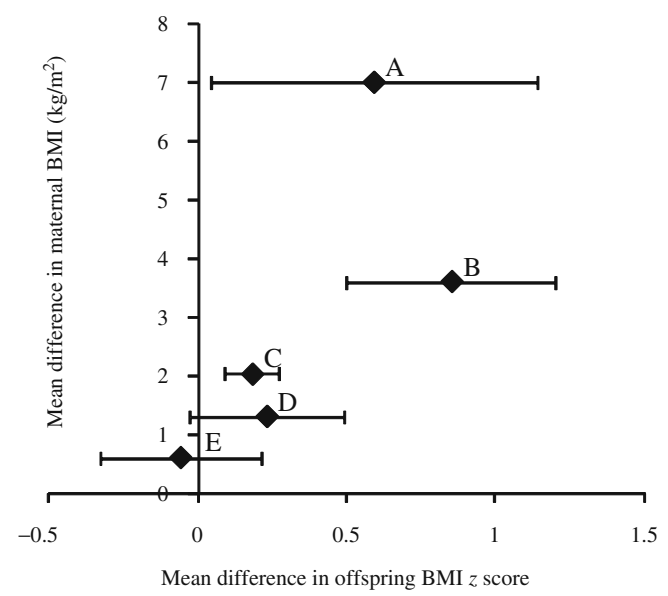

Fig. 6 The relationship between the difference in mean maternal BMI reported between diabetic and non-diabetic mothers and the difference between the mean BMI $z$ score of offspring of diabetic and non-diabetic mothers. Letters represent the following studies: A, Catalano et al. (2009) [29]; B, Krishnaveni et al. (2010) [22]; C, Gillman et al. (2003) [23]; D, Lawlor et al. (2009) [27]; E, Whitaker et al. (1998) [13]

An additional finding from our meta-analysis is that in the studies in which adjusted data $[15,27,46]$ were provided, the size of the observed difference in offspring BMI $z$ score between cases and controls was inversely proportional to the number of covariates included in the adjustment (see Table 1). This would indicate that maternal pre-pregnancy BMI is not the sole confounder and that other genetic and postnatal environmental factors may also be contributory [23].

Subgroup analysis suggests that the effects on offspring BMI are not influenced by type of maternal diabetes. This is in agreement with a previous study showing no difference in offspring outcome between mothers with type 1 diabetes and GDM [61]. However, given the small

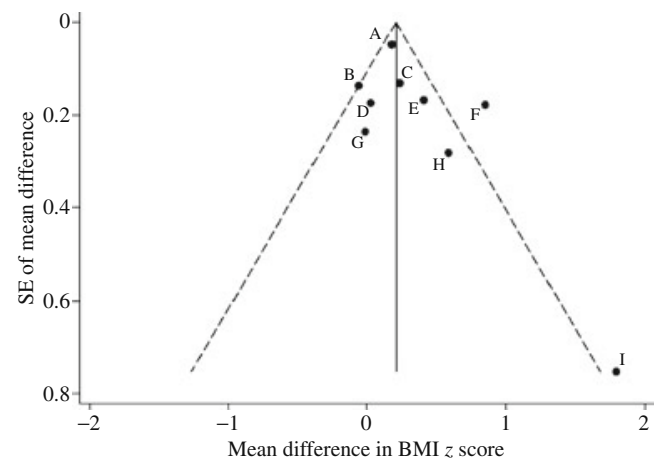

Fig. 7 Funnel plot of mean difference in offspring BMI $z$ score from mothers with diabetes and controls against standard errors, with $95 \%$ confidence limits. Pooled mean difference $=0.280$. Letters represent the following studies: A, Gillman et al. (2003) [23]; B, Whitaker et al. (1998) [13]; C, Lawlor et al. (2009) [27]; D, Wright et al. (2009) [46]; E, Lindsay et al. (2010) [15]; F, Krishnaveni et al. (2010) [22]; G, Manderson et al. (2002) [14]; H, Catalano et al. (2009) [29]; I, Hunter et al. (2004) [50]. Pooled mean difference $=0.280$ number of studies and participants, conclusions drawn from the subgroup analysis must be viewed with caution. There were insufficient data to perform a meta-analysis with regard to offspring of mothers with pre-pregnancy type 2 diabetes.

We acknowledge several limitations to the extent to which conclusions can be drawn from meta-analyses of observational studies with variable treatment of confounders and variable definition of GDM. Some studies used the National Diabetes Data Group [62] or Carpenter and Coustan criteria [63] and others, the clinical diagnosis reported in the medical records. The case-definition for diabetes has a significant effect on reported population prevalence of GDM, varying, for example, between 3.17\% and $4.48 \%$ according to the National Diabetes Data Group and Carpenter and Coustan criteria, respectively [64]. Inclusion of women with lesser degrees of glucose intolerance as diabetic would result in an underestimation of overall effect.

Treatment of diabetes during pregnancy (ultimately glycaemic control) may be an important determinant of offspring outcomes [65]. We reported data on the treatment received by the mothers included in this meta-analysis in Table 1, but insufficient information was available on glycaemic control for us to perform further meaningful analysis. However, treatment regimens were similar across the studies.

Accepted clinical management of maternal diabetes is directed at ameliorating short-term consequences by achieving good glucose control during pregnancy and treating immediate newborn complications. The short-term outcomes of diabetic pregnancies have improved substantially over the past decades [66], but the potential long-term consequences now pose serious threats to adult wellbeing and population health. In adults, the metabolic syndrome is increasing [67] and is a leading cause of mortality worldwide [68]. Similarly, in children, obesity and other features of the metabolic syndrome are rising rapidly and are strongly associated with premature death in later life [69].

We acknowledge that the number of included studies is small. Given the age range of the infants included, BMI $z$ score is the only possible way of including them in this meta-analysis. Several studies report outcomes in the form of obesity, relative body mass, BMI or weight. We contacted the authors of these studies, but not all were able to provide their results as BMI $z$ scores for inclusion in the meta-analysis. We would recommend that future studies on offspring of diabetic mothers during childhood should report their findings as BMI $z$ scores, together with other measures of weight and body composition. The use of standardised units will greatly facilitate future metaanalyses in this area. It also seems logical to suggest that the difference in offspring BMI $z$ score may increase with 
ageing, and a recent study suggests that this is the case [70]. However, given the overlap in age between the various reported cohorts, we felt that subgroup analyses attempting to address this possibility could be misleading.

Our meta-analysis of observational studies reveals an association between maternal diabetes and offspring BMI that is no longer significant after adjusting for maternal prepregnancy BMI. Thus, although diabetic control during pregnancy remains important, given the complex relationship between maternal BMI, maternal diabetes and offspring outcome, the importance of maintaining a healthy weight in women of childbearing age must also be emphasised.

Acknowledgements The authors would like to thank M. W. Gillman and G. V. Krishnaveni for contributing additional data to this meta-analysis.

Duality of interest S. Santhakumaran is funded by the National Institute of Health Research. No external funding was received specifically for this work. The authors declare that there is no duality of interest associated with this manuscript.

\section{References}

1. Bell R, Bailey K, Cresswell T, Hawthorne G, Critchley J, LewisBarned N (2008) Trends in prevalence and outcomes of pregnancy in women with pre-existing type I and type II diabetes. BJOG 115:445-452

2. Dabelea D, Snell-Bergeon JK, Hartsfield CL, Bischoff KJ, Hamman RF, McDuffie RS (2005) Increasing prevalence of gestational diabetes mellitus (GDM) over time and by birth cohort: Kaiser Permanente of Colorado GDM Screening Program. Diabetes Care 28:579-584

3. Freinkel N (1980) Banting Lecture 1980. Of pregnancy and progeny. Diabetes 29:1023

4. Freinkel N, Metzger BE (1978) Pregnancy as a tissue culture experience: the critical implications of maternal metabolism for fetal development. Ciba Found Symp pp 3-28

5. Clausen TD, Mathiesen ER, Hansen $T$ et al (2008) High prevalence of type 2 diabetes and pre-diabetes in adult offspring of women with gestational diabetes mellitus or type 1 diabetes. Diabetes Care 31:340

6. Simeoni U, Barker D (2009) Offspring of diabetic pregnancy: long-term outcomes. Semin Fetal Neonatal Med 14:119

7. Bihoreau MT, Ktorza A, Kinebanyan MF, Picon L (1986) Impaired glucose homeostasis in adult rats from hyperglycemic mothers. Diabetes 35:979-984

8. Franke K, Harder T, Aerts L et al (2005) 'Programming' of orexigenic and anorexigenic hypothalamic neurons in offspring of treated and untreated diabetic mother rats. Brain Res 1031:276

9. Pettitt DJ, Aleck KA, Baird HR, Carraher MJ, Bennett PH, Knowler WC (1988) Congenital susceptibility to NIDDM. Role of intrauterine environment. Diabetes 37:622

10. Dabelea D, Hanson RL, Lindsay RS et al (2000) Intrauterine exposure to diabetes conveys risks for type 2 diabetes and obesity: a study of discordant sibships. Diabetes 49:22082211

11. Fetita L-S, Sobngwi E, Serradas P, Calvo F, Gautier J-F (2006) Consequences of fetal exposure to maternal diabetes in offspring. J Clin Endocrinol Metab 91:3718
12. Meigs JB, Wilson PW, Fox CS et al (2006) Body mass index, metabolic syndrome, and risk of type 2 diabetes or cardiovascular disease. J Clin Endocrinol Metab 91:2906-2912

13. Whitaker RC, Pepe MS, Seidel KD, Wright JA, Knopp RH (1998) Gestational diabetes and the risk of offspring obesity. Pediatrics 101:E9

14. Manderson JG, Mullan B, Patterson CC, Hadden DR, Traub AI, McCance DR (2002) Cardiovascular and metabolic abnormalities in the offspring of diabetic pregnancy. Diabetologia 45:991-996

15. Lindsay RS, Nelson SM, Walker JD et al (2010) Programming of adiposity in offspring of mothers with type 1 diabetes at age 7 years. Diabetes Care 33:1080-1085

16. Stroup DF, Berlin JA, Morton SC et al (2000) Meta-analysis of observational studies in epidemiology: a proposal for reporting. JAMA 283:2008

17. Collaboration TC (2008) Review manager. The Nordic Cochrane Centre, Copenhagen

18. Altman DG, Bland JM (2003) Interaction revisited: the difference between two estimates. BMJ 326:219

19. Cochran WG (1954) The combination of estimates from different experiments. Biometrics 10:101-129

20. Higgins JP, Thompson SG (2002) Quantifying heterogeneity in a meta-analysis. Stat Med 21:1539-1558

21. Egger M, Smith GD, Schneider M, Minder C (1997) Bias in meta-analysis detected by a simple, graphical test. BMJ 315:629-634

22. Krishnaveni GV, Veena SR, Hill JC, Kehoe S, Karat SC, Fall CH (2010) Intrauterine exposure to maternal diabetes is associated with higher adiposity and insulin resistance and clustering of cardiovascular risk markers in Indian children. Diabetes Care $33: 402-404$

23. Gillman MW, Rifas-Shiman S, Berkey CS, Field AE, Colditz GA (2003) Maternal gestational diabetes, birth weight, and adolescent obesity. Pediatrics 111:e221-e226

24. Rodrigues S, Ferris AM, Perez-Escamilla R, Backstrand JR (1998) Obesity among offspring of women with type 1 diabetes. Clin Invest Med 21:258

25. Silverman BL, Landsberg L, Metzger BE (1993) Fetal hyperinsulinism in offspring of diabetic mothers. Association with the subsequent development of childhood obesity. Ann NY Acad Sci 699:36

26. Buinauskiene J, Baliutaviciene D, Zalinkevicius R (2004) Glucose tolerance of 2- to 5-yr-old offspring of diabetic mothers. Pediatr Diab 5:143

27. Lawlor DA, Fraser A, Lindsay RS et al (2010) Association of existing diabetes, gestational diabetes and glycosuria in pregnancy with macrosomia and offspring body mass index, waist and fat mass in later childhood: findings from a prospective pregnancy cohort. Diabetologia 53:89-97

28. Bunt JC, Tataranni PA, Salbe AD (2005) Intrauterine exposure to diabetes is a determinant of hemoglobin $\mathrm{A}(1) \mathrm{c}$ and systolic blood pressure in pima Indian children. J Clin Endocrinol Metab 90:3225-3229

29. Catalano P, Farrell K, Thomas A et al (2009) Perinatal risk factors for childhood obesity and metabolic dysregulation. Am J Clin Nutr 90:1303

30. Cho NH, Silverman BL, Rizzo TA, Metzger BE (2000) Correlations between the intrauterine metabolic environment and blood pressure in adolescent offspring of diabetic mothers. J Pediatr 136:587

31. Kostalova L, Leskova L, Kapellerova A, Strbak V (2001) Body mass, plasma leptin, glucose, insulin and C-peptide in offspring of diabetic and non-diabetic mothers. Eur J Endocrinol 145:53

32. Kvehaugen AS, Andersen LF, Staff AC (2010) Anthropometry and cardiovascular risk factors in women and offspring after 
pregnancies complicated by preeclampsia or diabetes mellitus. Acta Obstet Gynecol Scand 89:1478-1485

33. Malee MP, Verma A, Messerlian G, Tucker R, Vohr BR (2002) Association between maternal and child leptin levels 9 years after pregnancy complicated by gestational diabetes. Horm Metab Res 34:212

34. Mughal MZ, Eelloo J, Roberts SA et al (2010) Body composition and bone status of children born to mothers with type 1 diabetes mellitus. Arch Dis Child, England 95:281-285

35. Pettitt DJ, Knowler WC, Bennett PH, Aleck KA, Baird HR (1987) Obesity in offspring of diabetic Pima Indian women despite normal birth weight. Diabetes Care 10:76-80

36. Pettitt DJ, Knowler WC (1998) Long-term effects of the intrauterine environment, birth weight, and breast-feeding in Pima Indians. Diabetes Care 21(Suppl 2):B138-B141

37. Pirkola J, Vrsmki M, Leinonen E et al (2008) Maternal type 1 and gestational diabetes: postnatal differences in insulin secretion in offspring at preschool age. Pediatr Diab 9:583

38. Silverman BL, Rizzo T, Green OC et al (1991) Long-term prospective evaluation of offspring of diabetic mothers. Diabetes 40(Suppl 2):121-125

39. Silverman BL, Metzger BE, Cho NH, Loeb CA (1995) Impaired glucose tolerance in adolescent offspring of diabetic mothers. Relationship to fetal hyperinsulinism. Diabetes Care 18:611-617

40. Salbe AD, Lindsay RS, Collins CB, Tataranni PA, Krakoff J, Bunt JC (2007) Comparison of plasma insulin levels after a mixed-meal challenge in children with and without intrauterine exposure to diabetes. J Clin Endocrinol Metab 92:624-628

41. Tam W, Ma RCW, Yang X et al (2008) Glucose intolerance and cardiometabolic risk in children exposed to maternal gestational diabetes mellitus in utero. Pediatrics 122:1229

42. Tam WH, Ma RC, Yang X et al (2010) Glucose intolerance and cardiometabolic risk in adolescents exposed to maternal gestational diabetes: a 15-year follow-up study. Diabetes Care, United States 33:1382-1384

43. Touger L, Looker H, Krakoff J, Lindsay R, Cook V, Knowler W (2005) Early growth in offspring of diabetic mothers. Diabetes Care 28:585

44. Weiss PA, Scholz HS, Haas J, Tamussino KF, Seissler J, Borkenstein MH (2000) Long-term follow-up of infants of mothers with type 1 diabetes: evidence for hereditary and nonhereditary transmission of diabetes and precursors. Diabetes Care 23:905

45. Wroblewska-Seniuk K, Wender-Ozegowska E, Szczapa J (2009) Long-term effects of diabetes during pregnancy on the offspring. Pediatr Diab 10:432

46. Wright CS, Rifas-Shiman SL, Rich-Edwards JW, Taveras EM, Gillman MW, Oken E (2009) Intrauterine exposure to gestational diabetes, child adiposity, and blood pressure. Am J Hypertens 22:215-220

47. Silverman BL, Rizzo TA, Cho NH, Metzger BE (1998) Long-term effects of the intrauterine environment. The Northwestern University Diabetes in Pregnancy Center. Diabetes Care 21(Suppl 2):B142

48. Boerschmann H, Pfluger M, Henneberger L, Ziegler AG, Hummel S (2010) Prevalence and predictors of overweight and insulin resistance in offspring of mothers with gestational diabetes mellitus. Diabetes Care 33:1845-1849

49. Krishnaveni G, Hill J, Leary S et al (2005) Anthropometry, glucose tolerance, and insulin concentrations in Indian children: relationships to maternal glucose and insulin concentrations during pregnancy. Diabetes Care 28:2919

50. Hunter WA, Cundy T, Rabone D et al (2004) Insulin sensitivity in the offspring of women with type 1 and type 2 diabetes. Diabetes Care 27:1148-1152
51. Hummel S, Pfluger M, Kreichauf S, Hummel M, Ziegler AG (2009) Predictors of overweight during childhood in offspring of parents with type 1 diabetes. Diabetes Care 32:921-925

52. Lindsay RS, Hanson RL, Bennett PH, Knowler WC (2000) Secular trends in birth weight, BMI, and diabetes in the offspring of diabetic mothers. Diabetes Care 23:1249-1254

53. Deierlein AL, Siega-Riz AM, Chantala K, Herring AH (2011) The association between maternal glucose concentration and child BMI at age 3 years. Diabetes Care 34:480-484

54. Pirkola J, Pouta A, Bloigu A et al (2010) Risks of overweight and abdominal obesity at age 16 years associated with prenatal exposures to maternal prepregnancy overweight and gestational diabetes mellitus. Diabetes Care 33:1115

55. Van Assche FA, Holemans K, Aerts L (2001) Long-term consequences for offspring of diabetes during pregnancy. $\mathrm{Br}$ Med Bull 60:173

56. Metzger BE, Silverman BL, Freinkel N, Dooley SL, Ogata ES, Green OC (1990) Amniotic fluid insulin concentration as a predictor of obesity. Arch Dis Child 65:1050

57. Hillier T, Pedula K, Schmidt M, Mullen J, Charles M-A, Pettitt D (2007) Childhood obesity and metabolic imprinting: the ongoing effects of maternal hyperglycemia. Diabetes Care 30:2287

58. Boney C, Verma A, Tucker R, Vohr B (2005) Metabolic syndrome in childhood: association with birth weight, maternal obesity, and gestational diabetes mellitus. Pediatrics 115:e290

59. Aerts L, van Assche FA (2003) Intra-uterine transmission of disease. Placenta 24:905-911

60. Kalkhoff RK (1991) Impact of maternal fuels and nutritional state on fetal growth. Diabetes 40(Suppl 2):61

61. Plagemann A, Harder T, Kohlhoff R, Rohde W, Drner G (1997) Overweight and obesity in infants of mothers with long-term insulin-dependent diabetes or gestational diabetes. Int $\mathrm{J}$ Obes 21:451

62. National Diabetes Data Group (1979) Classification and diagnosis of diabetes mellitus and other categories of glucose intolerance. Diabetes 28:1039-1057

63. Carpenter MW, Coustan DR (1982) Criteria for screening tests for gestational diabetes. Am J Obstet Gynecol 144:768-773

64. Karcaaltincaba D, Kandemir O, Yalvac S, Guvendag-Guven S, Haberal A (2009) Prevalence of gestational diabetes mellitus and gestational impaired glucose tolerance in pregnant women evaluated by National Diabetes Data Group and Carpenter and Coustan criteria. Int J Gynaecol Obstet 106:246-249

65. Metzger BE, Lowe LP, Dyer AR et al (2008) Hyperglycemia and adverse pregnancy outcomes. N Engl J Med 358:1991-2002

66. Rijpert M, Evers I, de Vroede MAMJ, de Valk H, Heijnen C, Visser GHA (2009) Risk factors for childhood overweight in offspring of type 1 diabetic women with adequate glycemic control during pregnancy: nationwide follow-up study in the Netherlands. Diabetes Care 32:2099

67. Ford ES, Giles WH, Mokdad AH (2004) Increasing prevalence of the metabolic syndrome among U.S. adults. Diabetes Care 27:2444-2449

68. Malik S, Wong ND, Franklin SS et al (2004) Impact of the metabolic syndrome on mortality from coronary heart disease, cardiovascular disease, and all causes in United States adults. Circulation 110:1245-1250

69. Franks PW, Hanson RL, Knowler WC, Sievers ML, Bennett PH, Looker HC (2010) Childhood obesity, other cardiovascular risk factors, and premature death. N Engl J Med 362:485-493

70. Baptiste-Roberts K, Nicholson WK, Wang NY, Brancati FL (2011) Gestational diabetes and subsequent growth patterns of offspring: The National Collaborative Perinatal Project. Matern Child Health J doi:10.1007/s10995-011-0756-2 